\title{
O’Geen, S1 Fig
}

\section{$\underline{\mathrm{h} N \mathrm{AAgO}}$}

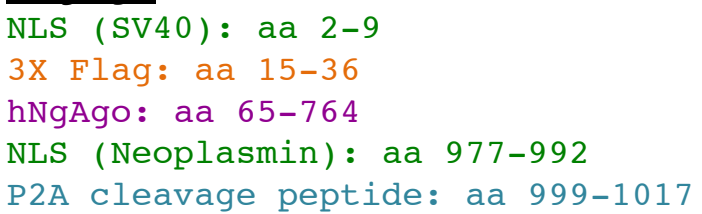

MPKKKRKVGGSGGSDYKDHDGDYKDHDIDYKDDDDKGGGSGGGSGTGGSGGSGGSGGSGGSGRPMTVIDLDSTTTADE LTSGHTYDISVTLTGVYDNTDEQHPRMSLAFEQDNGERRYITLWKNTTPKDVFTYDYATGSTYIFTNIDYEVKDGYEN LTATYQTTVENATAQEVGTTDEDETFAGGEPLDHHLDDALNETPDDAETESDSGHVMTSFASRDQLPEWTLHTYTLTA TDGAKTDTEYARRTLAYTVRQELYTDHDAAPVATDGLMLLTPEPLGETPLDLDCGVRVEADETRTLDYTTAKDRLLAR ELVEEGLKRSLWDDYLVRGIDEVLSKEPVLTCDEFDLHERYDLSVEVGHSGRAYLHINFRHRFVPKLTLADIDDDNIY PGLRVKTTYRPRRGH IVWGLRDECATDSLNTLGNQSVVAYHRNNQTPINTDLLDAIEAADRRVVETRRQGHGDDAVSF PQELLAVEPNTHQIKQFASDGF HQQARSKTRLSASRCSEKAQAFAERLDPVRLNGSTVEFSSEFFTGNNEQQLRLLYE NGESVLTFRDGARGAHPDETFSKGIVNPPESFEVAVVLPEQQADTCKAQWDTMADLLNQAGAPPTRSETVQYDAFSSP ESISLNVAGAIDPSEVDAAFVVLPPDQEGFADLASPTETYDELKKALANMGIYSOMAYFDRFRDAKIFYTRNVALGLL AAAGGVAFTTEHAMPGDADMF IGIDVSRSYPEDGASGQINIAATATAVYKDGTILGHSSTRPQLGEKLQSTDVRDIMK NAILGYQQVTGESPTH IVIHRDGFMNEDLDPATEFLNEQGVEYDIVEIRKQPQTRLLAVSDVQYDTPVKSIAAINQNE PRATVATFGAPEYLATRDGGGLPRPIQIERVAGETDIETLTRQVYLLSQSHIQVHNSTARLPITTAYADQASTHATKG YLVQTGAFESNVGFLGGSGGSGGSGGSGGSASGGGSGGGSKRPAATKKAGQAKKKKGGSGSGATNFSLLKOAGDVEEN PGPAAA

\section{$\underline{\text { htAgo }}$}

NLS (SV40): aa 2-9

3X Flag: aa 15-36

hTtAgo: aa 65-749

NLS (Neoplasmin): aa 775-790

P2A cleavage peptide: aa 797-815

MPKKKRKVGGSGGSDYKDHDGDYKDHDIDYKDDDDKGGGSGGGSGTGGSGGSGGSGGSGGSGRPMNHLGKTEVFLNRF ALRPLNPEELRPWRLEVVLDPPPGREEVYPLLAOVARRAGGVTVRMGDGLASWSPPEVLVLEGTLARMGQTYAYRLYP KGRRPLDPKDPGERSVLSALARRLLQERLRRLEGVWVEGLAVYRREHARGPGWRVLGGAVLDLWVSDSGAFLLEVDPA YRILCEMSLEAWLAQGHPLPKRVRNAYDRRTWELLRLGEEDPKELPLPGGLSLLDYHASKGRLQGREGGRVAWVADPK DPRKPIPHLTGLLVPVLTLEDLHEEEGSLALSLPWEERRRRTREIASWIGRRLGLGTPEAVRAQAYRLS IPKLMGRRA VSKPADALRVGFYRAQETALALLRLDGAQGWPEFLRRALLRAFGASGASLRLHTLHAHPSQGLAFREALRKAKEEGVQ AVLVLTPPMAWEDRNRLKALLLREGLPSQILNVPLREEERHRWENALLGLLAKAGLQVVALSGAYPAELAVGFDAGGR ESFRFGGAACAVGGDGGHLLWTLPEAQAGERIPQEVVWDLLEETLWAFRRKAGRLPSRVLLLRDGRVPQDEFALALEA LAREGIAYDLVSVRKSGGGRVYPVQGRLADGLYVPLEDKTFLLLTVHRDFRGTPRPLKLVHEAGDTPLEALAHQIFHL TRLYPASGFAFPRLPAPLHLADRLVKEVGRLGIRHLKEVDREKLFFVGGSGGSGGSGGSGGSASGGGSGGGSKRPAAT KKAGQAKKKKGGSGSGATNF SLLKQAGDVEENPGPAAA

\section{S1 Fig. Protein sequences of $\mathrm{hNgAgo}$ and $\mathrm{hTtAgo}$ used in this study.}

